\title{
A computer test of holographic flavour dynamics. Part II
}

\author{
Yuhma Asano, Veselin G. Filev, Samuel Kováčik and Denjoe O'Connor \\ School of Theoretical Physics, Dublin Institute for Advanced Studies, \\ 10 Burlington Road, Dublin 4, Ireland \\ E-mail: yuhma@stp.dias.ie, vfilev@stp.dias.ie, skovacik@stp.dias.ie, \\ denjoe@stp.dias.ie
}

AbStract: We study the second derivative of the free energy with respect to the fundamental mass (the mass susceptibility) for the Berkooz-Douglas model as a function of temperature and at zero mass. The model is believed to be holographically dual to a D0/D4 intersection. We perform a lattice simulation of the system at finite temperature and find excellent agreement with predictions from the gravity dual.

Keywords: AdS-CFT Correspondence, D-branes, Lattice Quantum Field Theory, $\mathrm{M}$ (atrix) Theories

ARXiv EPrint: 1612.09281 


\section{Contents}

1 Introduction 1

2 Mass susceptibility of the condensate at high temperature 2

3 Holographic description at low temperature 3

4 Lattice results $\quad 5$

5 Conclusion $\quad 6$

$\begin{array}{lll}\text { A Analytic expression for the condensate susceptibility } & 8\end{array}$

\section{Introduction}

Gauge/gravity duality [1,2], the idea that gravity can capture the dynamics of strongly coupled gauge theories and vice versa continues to fascinate theoretical physicists. Numerous applications ranging from condensed matter physics to heavy ion collisions have been proposed. Most of them exploit the weak/strong coupling duality of the correspondence. However, it is this property that makes the correspondence difficult to test, especially in a non-supersymmetric setting. In this paper we continue recent efforts to test a particular regime of the correspondence at finite temperature using mainly lattice simulations [3-9]. We focus on the Berkooz-Douglas (BD) matrix model [10], a flavoured version of the BFSS matrix model [11], holographically dual to the D0/D4 system [12-14].

In ref. [15] the lattice formulation of the BD matrix model was studied. The model was studied both holographically and with computer simulations focusing on the fundamental condensate of the theory as a main observable. In the large $N$ limit, as the mass parameter is varied, gauge/gravity duality predicts the existence of a meson melting phase transition, corresponding to a topology change transition in the supergravity set-up. The studies of ref. [15] show a remarkable agreement between theory and simulations in the deconfined phase of the theory. It was speculated that in this phase there is a cancellation mechanism for the $\alpha^{\prime}$ corrections to the condensate. The studies were conducted at two different temperatures and for a variety of bare masses.

In this paper we consider the opposite regime studying the susceptibility of the condensate with respect to the bare mass, at vanishing bare mass and for a range of different temperatures. The advantage of this approach is that at high temperatures the BD model can be studied perturbatively [16] and at low temperatures we have a gauge/gravity prediction. This allows us at high temperatures to verify the validity of our lattice approach against the perturbative results of ref. [16] while at low temperatures to compare with the 
predictions of gauge/gravity duality. Furthermore, we find that if we go to sufficiently high order in perturbation theory we can extrapolate the high temperature expansion to intermediate temperatures. If the cancellation mechanism for the $\alpha^{\prime}$ corrections to the fundamental condensate does take place we may expect to obtain agreement of these extrapolated high temperature results with the gauge/gravity curve. Remarkably, the low temperature curve obtained from the D0/D4 holographic set-up and the high temperature expansion curves are indeed very close in the intermediate temperature regime $T \sim \lambda^{1 / 3}$.

The paper is organised as follows. In section 2 , we briefly review the lattice formulation of the BD matrix model and its high temperature expansion. In section 3 , with details in appendix A, we present the derivation of the slope of the condensate from supergravity. In section 4 , we present our results for the slope of the fundamental condensate. We conclude with a discussion in section 5 .

\section{Mass susceptibility of the condensate at high temperature}

The $\mathrm{BD}$ model in euclidean $1+0$ dimensions is given by the following action [15-17]:

$$
\begin{aligned}
S_{E}=N \int_{0}^{\beta} d \tau[ & \operatorname{Tr}\left(\frac{1}{2} D_{\tau} X^{a} D_{\tau} X^{a}+\frac{1}{2} D_{\tau} \bar{X}^{\rho \dot{\rho}} D_{\tau} X_{\rho \dot{\rho}}+\frac{1}{2} \lambda^{\dagger \rho} D_{\tau} \lambda_{\rho}+\frac{1}{2} \theta^{\dagger \dot{\rho}} D_{\tau} \theta_{\dot{\rho}}\right) \\
& +\operatorname{tr}\left(D_{\tau} \bar{\Phi}^{\rho} D_{\tau} \Phi_{\rho}+\chi^{\dagger} D_{\tau} \chi\right) \\
& -\operatorname{Tr}\left(\frac{1}{4}\left[X^{a}, X^{b}\right]^{2}+\frac{1}{2}\left[X^{a}, \bar{X}^{\rho \dot{\rho}}\right]\left[X^{a}, X_{\rho \dot{\rho}}\right]\right) \\
& +\frac{1}{2} \operatorname{Tr} \sum_{A=1}^{3} \mathcal{D}^{A} \mathcal{D}^{A}+\operatorname{tr}\left(\bar{\Phi}^{\rho}\left(X^{a}-m^{a}\right)^{2} \Phi_{\rho}\right) \\
& -\operatorname{Tr}\left(-\frac{1}{2} \lambda^{\dagger \rho} \gamma^{a}\left[X^{a}, \lambda_{\rho}\right]+\frac{1}{2} \theta^{\dagger \dot{\rho}} \gamma^{a}\left[X^{a}, \theta_{\dot{\rho}}\right]-\sqrt{2} i \varepsilon^{\rho \sigma} \theta^{\dagger \dot{\rho}}\left[X_{\sigma \dot{\rho}}, \lambda_{\rho}\right]\right) \\
& \left.-\operatorname{tr}\left(\chi^{\dagger} \gamma^{a}\left(X^{a}-m^{a}\right) \chi+\sqrt{2} i \varepsilon^{\rho \sigma} \chi^{\dagger} \lambda_{\rho} \Phi_{\sigma}+\sqrt{2} i \varepsilon_{\rho \sigma} \bar{\Phi}^{\rho} \lambda^{\dagger \sigma} \chi\right)\right]
\end{aligned}
$$

where

$$
\mathcal{D}^{A}=\sigma_{\rho}^{A \sigma}\left(\frac{1}{2}\left[\bar{X}^{\rho \dot{\rho}}, X_{\sigma \dot{\rho}}\right]-\Phi_{\sigma} \bar{\Phi}^{\rho}\right)
$$

and the covariant derivative $D_{\tau}$ acts on the fields of the fundamental multiplet, $\Phi_{\rho}$ and $\chi$, as $D_{\tau} \cdot=\left(\partial_{\tau}-i A\right) \cdot$. The trace of the colour $\mathrm{SU}(N)$ is written as $\operatorname{Tr}$ while that of the flavour $\mathrm{SU}\left(N_{f}\right)$ is denoted by tr. The diagonal matrices, $m^{a}$, correspond to the transverse positions of the D4-branes.

Note that the overall factor of $N$ in equation (2.1) implies that the dimensionless parameter $\beta$ is related to the temperature via: $\beta^{-1}=T / \lambda^{1 / 3}$, where $\lambda=N g^{2}$ is the 't Hooft coupling. In the same way the dimensionless parameter $m^{a}=m_{q}^{a} / \lambda^{1 / 3}$, where $m_{q}^{a}$ is the bare mass in physical units. 
The fundamental condensate is defined as the variation of the free energy density with respect to the bare mass parameter $m^{a}$ :

$$
\left\langle\mathcal{O}_{m}^{a}\right\rangle=\frac{\partial}{\partial m^{a}}\left(-\frac{1}{\beta} \log Z\right)=\left\langle\frac{\partial}{\partial m^{a}} \frac{S_{E}}{\beta}\right\rangle=\left\langle\frac{N}{\beta} \int_{0}^{\beta} d \tau \operatorname{tr}\left\{2 \bar{\Phi}^{\rho}\left(m^{a}-X^{a}\right) \Phi_{\rho}+\chi^{\dagger} \gamma^{a} \chi\right\}\right\rangle .
$$

In this paper we focus on the mass susceptibility of the condensate at vanishing mass. The mass susceptibility of the condensate is, by definition,

$$
\left\langle\mathcal{C}^{m}\right\rangle=\frac{\partial^{2}}{\partial m^{a}}\left(-\frac{1}{\beta} \log Z\right)=\frac{1}{\beta}\left\langle\frac{\partial^{2} S_{E}}{\partial m^{a^{2}}}-\left(\frac{\partial S_{E}}{\partial m^{a}}\right)^{2}\right\rangle+\frac{1}{\beta}\left\langle\frac{\partial S_{E}}{\partial m^{a}}\right\rangle^{2},
$$

which can be written as

$$
\left\langle\mathcal{C}^{m}\right\rangle=\left\langle\partial_{m^{a}} \mathcal{O}_{m}^{a}\right\rangle-\beta\left\langle\left(\mathcal{O}_{m}^{a}\right)^{2}-\left\langle\mathcal{O}_{m}^{a}\right\rangle^{2}\right\rangle
$$

using $\mathcal{O}_{m}^{a}=\partial S_{E} / \partial m^{a}$. Now if we substitute $S_{E}$ with the action (2.1), for the operator $\mathcal{C}^{m}$ we obtain ${ }^{1}$

$$
\mathcal{C}^{m}=\frac{2 N}{\beta} \int_{0}^{\beta} d \tau \operatorname{tr} \bar{\Phi}^{\rho} \Phi_{\rho}-\frac{N^{2}}{\beta}\left(\int_{0}^{\beta} d \tau \operatorname{tr}\left\{-2 \bar{\Phi}^{\rho} X^{a} \Phi_{\rho}+\chi^{\dagger} \gamma^{a} \chi\right\}\right)^{2} .
$$

Recently, the high temperature expansion of the BD model was considered in ref. [16] using expansion in Matsubara modes and standard perturbation theory. The following expansion of the mass susceptibility was obtained:

$$
\frac{\left\langle\mathcal{C}^{m}\right\rangle}{N}=\beta^{-\frac{1}{2}} \Xi_{6}+\beta\left(\Xi_{7}+\Xi_{8}\right)+O\left(\beta^{\frac{5}{2}}\right)
$$

where the constants $\Xi_{6}, \Xi_{7}$ and $\Xi_{8}$ can be measured by simulating the pure matrix model obtained in the $T \rightarrow \infty$ limit and have been tabulated in ref. [16]. We can now use the lattice formulation of the BD model proposed in ref. [15] to compare to the high temperature expansion formula (2.7). Before we continue with the lattice studies of the susceptibility let us focus on the holographic description of the model at low temperature.

\section{Holographic description at low temperature}

At low temperature the BD model is proposed to be dual to the D0/D4 holographic set-up. ${ }^{2}$ The most understood case that we will focus on is the so called quenched approximation,

\footnotetext{
${ }^{1}$ Note that the expression for $\mathcal{C}^{m}$ in ref. [16] differs by a factor of $N$. Note also that the factor of $1 / 5$ in the reference compensates the summation over $a=1, \ldots, 5$ while, in this paper, $a$ is not summed over in $(2.4),(2.5)$ and $(2.6)$.

${ }^{2}$ The D0/D4 set-up belongs to a large class of $\mathrm{Dp} / \mathrm{Dp}+4$-brane intersections exhibiting universal properties such as the presence of a meson melting phase transition. For more details look at refs. [13, 14, 18-21] as well as ref. [22] for an extensive review.
} 
when the flavour D4-branes are in the probe approximation [12]. In the near horizon limit the D0-brane supergravity background is given by

$$
\begin{aligned}
d s^{2} & =-H^{-\frac{1}{2}} f d t^{2}+H^{\frac{1}{2}}\left(\frac{d u^{2}}{f}+u^{2} d \Omega_{8}^{2}\right), \\
e^{\Phi} & =H^{\frac{3}{4}}, \quad C_{0}=H^{-1},
\end{aligned}
$$

where $H=(L / u)^{7}$ and $f(u)=1-\left(u_{0} / u\right)^{7}$. Here $u_{0}$ is the radius of the horizon related to the Hawking temperature via $T=7 /(4 \pi L)\left(u_{0} / L\right)^{5 / 2}$ and the length scale $L$ is given by $L^{7}=15 / 2\left(2 \pi \alpha^{\prime}\right)^{5} \lambda$, with $\lambda$ the 't Hooft coupling.

To introduce matter in the fundamental representation we consider the addition of $N_{f}$ probe D4-branes. In the probe approximation $N_{f} \ll N$, their dynamics is governed by the Dirac-Born-Infeld action:

$$
S_{\mathrm{DBI}}=-\frac{N_{f}}{(2 \pi)^{4} \alpha^{15 / 2} g_{s}} \int d^{4} \xi e^{-\Phi} \sqrt{-\operatorname{det}\left\|G_{\alpha, \beta}+\left(2 \pi \alpha^{\prime}\right) F_{\alpha, \beta}\right\|},
$$

where $G_{\alpha, \beta}$ is the induced metric and $F_{\alpha, \beta}$ is the $\mathrm{U}(1)$ gauge field of the D4-brane, which we will set to zero. Parametrising the unit $S^{8}$ in the metric (3.1) as

$$
d \Omega_{8}^{2}=d \theta^{2}+\cos ^{2} \theta d \Omega_{3}^{2}+\sin ^{2} \theta d \Omega_{4}^{2}
$$

and taking a D4-brane embedding extended along $t, u, \Omega_{3}$ with a non-trivial profile $\theta(u)$, we obtain (after Wick rotation)

$$
S_{\mathrm{DBI}}^{E}=\frac{N_{f} \beta}{8 \pi^{2} \alpha^{\prime 5 / 2} g_{s}} \int d u u^{3} \cos ^{3} \theta(u) \sqrt{1+u^{2} f(u) \theta^{\prime}(u)^{2}} .
$$

The embedding extremising the action (3.4) can be obtain by solving numerically the corresponding non-linear equation of motion. The AdS/CFT dictionary then relates the behaviour of the solution at large radial distance $u$ to the bare mass and condensate of the theory via $[12-14]$

$$
\sin \theta=\frac{\tilde{m}}{\tilde{u}}+\frac{\tilde{c}}{\tilde{u}^{3}}+\ldots
$$

where $\tilde{u}=u / u_{0}$ and the parameters $\tilde{m}$ and $\tilde{c}$ are proportional to the bare mass and condensate of the theory. Therefore, the mass susceptibility of the condensate at zero bare mass $\left\langle\mathcal{C}^{m}\right\rangle$ is proportional to

$$
\left\langle\mathcal{C}^{m}\right\rangle \propto-\left.\left(\frac{d \tilde{c}}{d \tilde{m}}\right)\right|_{\tilde{m}=0}=\frac{7 \pi}{2} \frac{\csc (\pi / 7) \Gamma(3 / 7) \Gamma(5 / 7)}{\Gamma(1 / 7)^{2} \Gamma(2 / 7) \Gamma(4 / 7)} .
$$

The last expression was obtained by using that small $\tilde{m}$ implies small $\theta$, and hence the equation of motion for $\theta$ can be linearised and solved analytically. We refer the reader to appendix A for more details. Combining equation (3.6) with the exact expressions for the mass and condensate in terms of $\tilde{m}$ and $\tilde{c}$ [13-15]:

$$
\begin{aligned}
m & =m_{q} / \lambda^{1 / 3}=\frac{u_{0} \tilde{m}}{2 \pi \alpha^{\prime}}=\left(\frac{120 \pi^{2}}{49}\right)^{1 / 5}\left(\frac{T}{\lambda^{1 / 3}}\right)^{2 / 5} \tilde{m}, \\
\left\langle\mathcal{O}_{m}\right\rangle & =-\frac{N_{f} u_{0}^{3}}{2 \pi g_{s} \alpha^{\prime 3 / 2}} \tilde{c}=\left(\frac{2^{4} 15^{3} \pi^{6}}{7^{6}}\right)^{1 / 5} N_{f} N_{c}\left(\frac{T}{\lambda^{1 / 3}}\right)^{6 / 5}(-2 \tilde{c}),
\end{aligned}
$$


we obtain

$$
\left\langle\mathcal{C}^{m}\right\rangle=14^{1 / 5} 15^{2 / 5} \pi^{9 / 5} \frac{\csc (\pi / 7) \Gamma(3 / 7) \Gamma(5 / 7)}{\Gamma(1 / 7)^{2} \Gamma(2 / 7) \Gamma(4 / 7)} N_{f} N_{c}\left(\frac{T}{\lambda^{1 / 3}}\right)^{4 / 5} \approx 1.136 N_{f} N_{c}\left(\frac{T}{\lambda^{1 / 3}}\right)^{4 / 5} .
$$

Equation (3.8) is the holographic prediction for the mass susceptibility of the fundamental condensate, which in the next section we are going to test on the lattice.

\section{Lattice results}

In this section we use the lattice formulation of the BD model developed in ref. [15] to test both the high temperature expansion curve (2.7) and the holographic prediction (3.8). Remarkably, the two curves are already very close in the intermediate $T / \lambda^{1 / 3} \sim 1$ temperature regime (see figure 1) suggesting that the $\alpha^{\prime}$ corrections to the mass susceptibility are small.

As was shown in section 2, if we start with the action $S_{E}$ in equation (2.1) we arrive at equations (2.5) and (2.6). However on the lattice we have to substitute the corresponding lattice action $S_{\text {bos }}+S_{\text {ps.f }}$ [15] for $S_{E}$, where $S_{\text {bos }}$ is the discretised bosonic action and $S_{\text {ps.f }}$ is the pseudo-fermionic action, in which the fermions are represented (modulo a neglected phase) by pseudo-fermionic bosonic fields and a complicated fermionic matrix [15]. As a result equation (2.6) is no longer valid; however equation (2.5) remains valid, provided one substitutes the condensate operator $\mathcal{O}_{m}^{a}$ with the corresponding lattice operator, obtained by differentiating the lattice action with respect to the mass parameter $m^{a}$. In addition, the first term in equation (2.5) involves a further derivative with respect to $m^{a}$, which complicates the analysis due to the more complex mass dependence of the pseudo-fermionic action. $^{3}$ Nevertheless, the fact that the second term in (2.5) is the variance of the condensate operator remains true on the lattice. This means that we can use the simulation data for the calculation of the condensate to measure the variance term while the first term in (2.5) has to be calculated directly as an expectation value.

The computation of the condensate susceptibility is a nice consistency check of our codes, since it involves also the second momentum of the simulation data. We used this method for temperatures in the range $1 \leq T / \lambda^{1 / 3} \leq 5$. For temperatures $T<\lambda^{1 / 3}$, we found that the critical slowing down, related to the absence of a gap in the supersymmetric system, impedes the estimation of the variance. This is why for such low temperatures we used that the condensate is, to a very good approximation, linear near $m=0$ and since it vanishes at $m=0$ one can approximate

$$
\left\langle\mathcal{C}^{m}\right\rangle \approx\left\langle\mathcal{O}_{m}^{a}\right\rangle / m^{a}
$$

for small $m^{a}$. This method also has the advantage that it imposes the vanishing of the condensate at vanishing mass (which is true by symmetry) and lowers the numerical error. We used this method to estimate the slope at temperature $T=0.8 \lambda^{1 / 3}$.

\footnotetext{
${ }^{3}$ We refer the reader to ref. [15] for more details on the differentiation of the pseudo-fermionic action $S_{\text {ps.f. }}$.
} 
In our simulations we used lattice points out of $\Lambda=16,24,32,48$ for temperatures $T / \lambda^{1 / 3} \lesssim 1$. At higher temperatures (in the range $1 \lesssim T / \lambda^{1 / 3} \leq 4$ ) we lowered $\Lambda$ to preserve roughly the same lattice spacing. For temperatures higher than $T=4 \lambda^{1 / 3}$ we used $\Lambda=4$ lattice points. The rank of the gauge group was fixed at $N=10$ and we used one family of flavours $N_{f}=1$ to minimise the ratio $N_{f} / N$ and improve the probe approximation. Note that although on the gravity side we used the probe approximation, the lattice simulation was dynamical [15]. Finally, for our parameters the high temperature curve (2.7) is given by [16]

$$
\left\langle\mathcal{C}^{m}\right\rangle=14.08\left(\frac{T}{\lambda^{1 / 3}}\right)^{1 / 2}-3.02\left(\frac{T}{\lambda^{1 / 3}}\right)^{-1}+O\left(T^{-\frac{5}{2}}\right) .
$$

In figure 1 we present our main result. The red dashed curve is the holographic curve (3.8), and the black dashed curve is the high temperature curve (4.2). The blue bars represent the lattice simulations based on the lattice formulation developed in ref. [15] while the red bars correspond to independent lattice simulations based on a different lattice discretisation. ${ }^{4}$ The results of these simulations agree very well. The red error bar at $T=\lambda^{1 / 3}$ has been obtained by extrapolating to $\Lambda=\infty$ using simulations with $\Lambda=16,24,32$ and 48 (see figure 2). The extrapolated result of $\left.\left\langle\mathcal{C}^{m}\right\rangle\right|_{T=\lambda^{1 / 3}}$ by a linear function is $11.26 \pm 0.29$ and the one by a quadratic function is $11.33 \pm 0.57$; they perfectly agree with the AdS/CFT prediction (3.8) for $T=\lambda^{1 / 3}$ and $N=10:\left.\left\langle\mathcal{C}^{m}\right\rangle\right|_{T=\lambda^{1 / 3}}=11.36$.

Overall, one can observe excellent agreement of the lattice simulation and the high temperature curve even for temperatures as low as $T=\lambda^{1 / 3}$. One can also observe excellent agreement with holographic predictions at temperatures $T \sim \lambda^{1 / 3}$. Remarkably, even the high temperature curve is very close to the holographic curve in this regime. As mentioned earlier this suggests that the $\alpha^{\prime}$ corrections to the mass susceptibility are indeed very small.

\section{Conclusion}

In this paper we continue our investigation of the BD model and its relation to the D0/D4brane holographic set-up. The main observable that we consider is the mass susceptibility of the fundamental condensate at vanishing fundamental mass. We applied the recent analysis of the high temperature regime of the $\mathrm{BD}$ matrix model to write down a perturbative expression for the susceptibility at high temperatures. We also review the holographic derivation of the fundamental condensate and obtain an analytic result for the susceptibility valid at low temperatures. Based on the observation of ref. [15] that the $\alpha^{\prime}$ corrections to the condensate seem to be insignificant in the deconfined phase (which is the relevant phase at vanishing bare mass) we expect to find good agreement with the holographic curve not only at very low temperatures, but also at intermediate temperatures $T \leq \lambda^{1 / 3}$. Remarkably, the high temperature expansion curve also remains valid down to intermediate temperatures $T \geq \lambda^{1 / 3}$ and is in fact very close to the AdS/CFT curve. Our lattice simulation is also in excellent agreement with both the high temperature and low temperature predictions, verifying the validity of the gauge/gravity correspondence.

\footnotetext{
${ }^{4}$ The latter lattice simulations use lattice derivatives in the second-order method [23].
} 


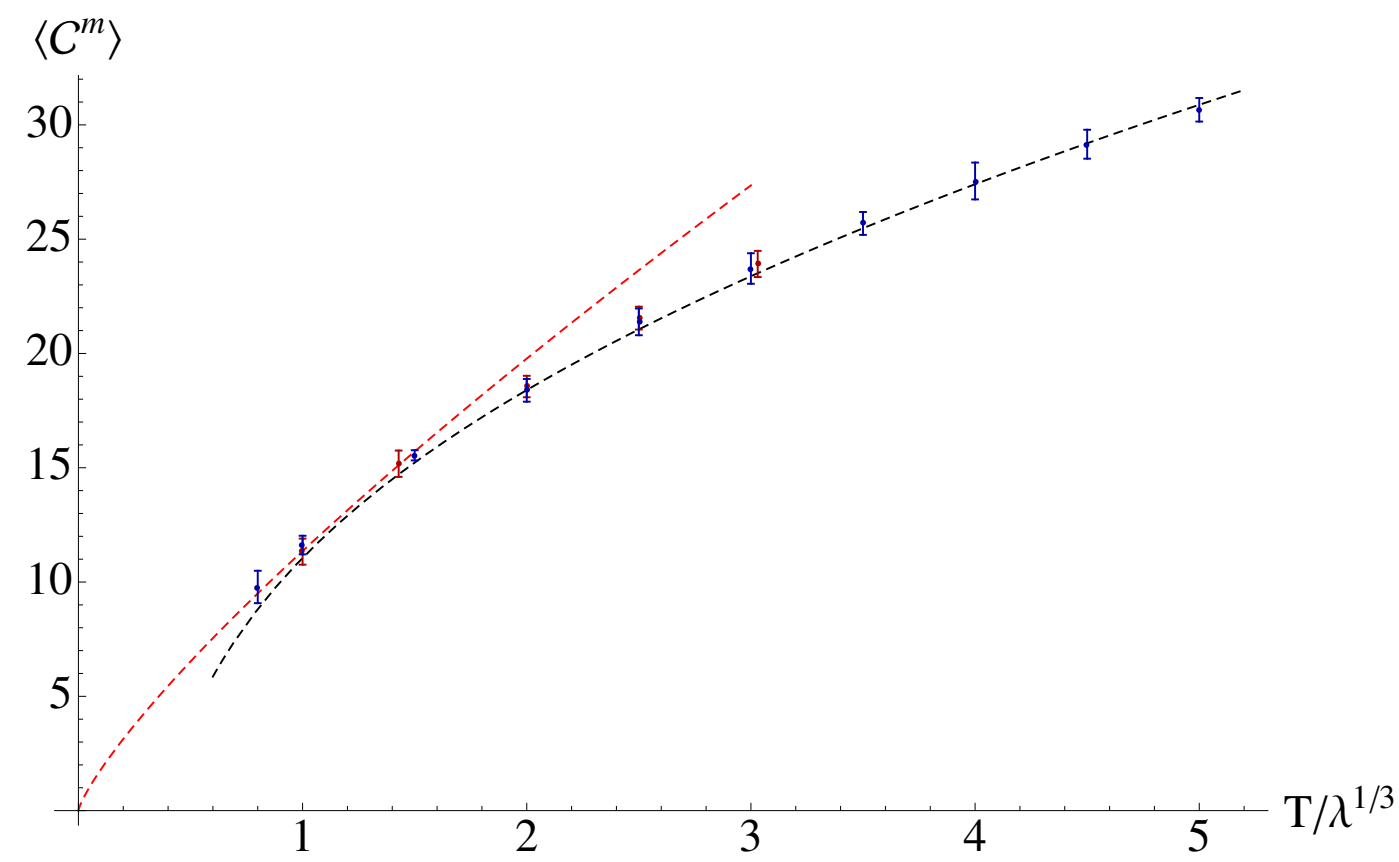

Figure 1. The red curve represents the holographic prediction (3.8), while the black dashed curve corresponds to the high temperature expansion curve (4.2). The blue bars represent the results of lattice simulations using the lattice discretisation in ref. [15]. The red bars correspond to independent lattice simulations based on a different lattice discretisation.

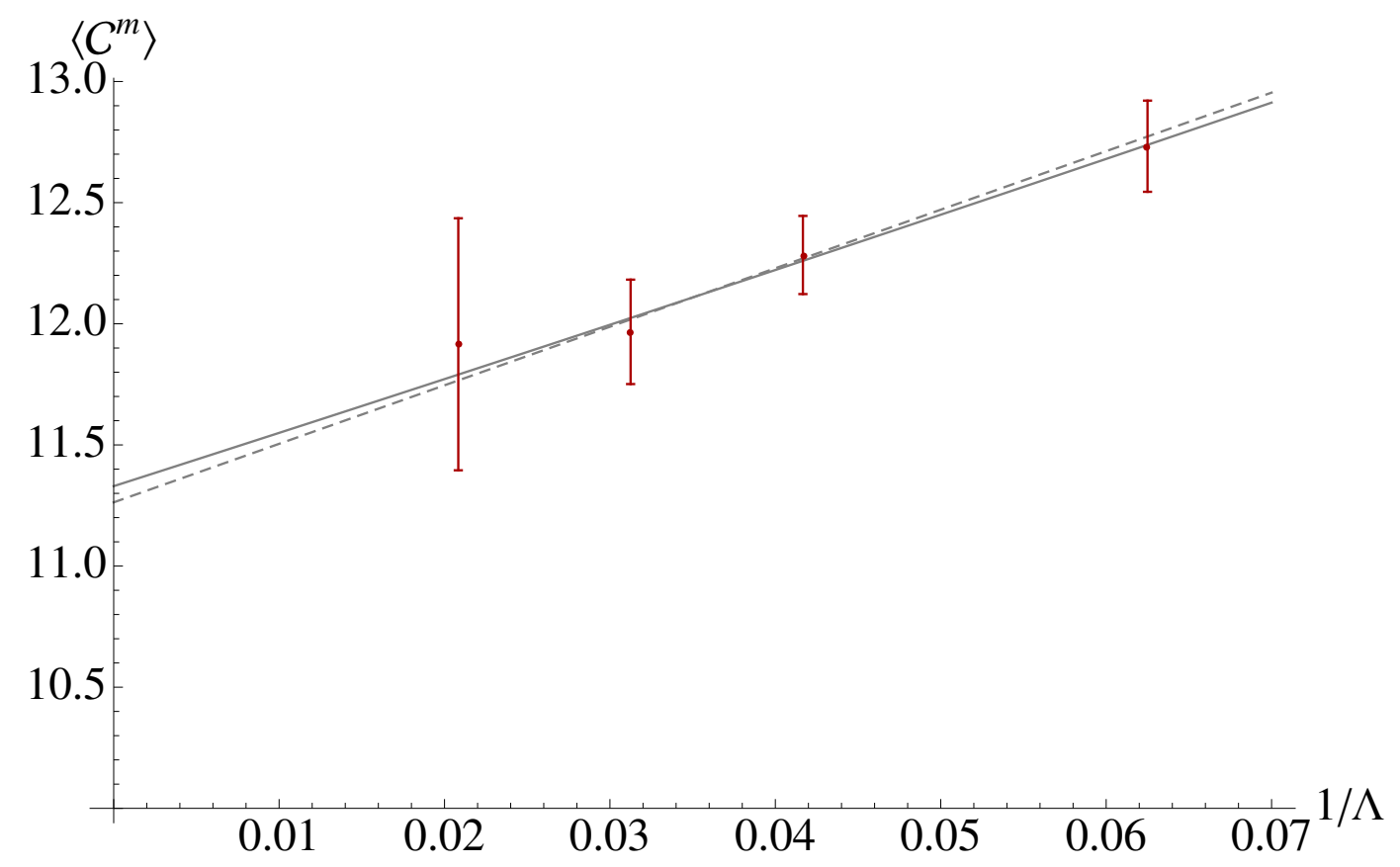

Figure 2. The red bars correspond to measurements at $\Lambda=16,24,32$ and 48 for $T=\lambda^{1 / 3}$ and $N=10$. The dashed gray line represents the linear extrapolation, and the solid gray curve corresponds to the quadratic extrapolation. 
Our results can be extended in several directions. The numerical direction is to push the simulation to lower temperatures, higher rank gauge groups (larger $N$ ) and larger $\Lambda$, the number of lattice points. The theoretical direction is to try to extend the validity of the high temperature curve by considering higher order perturbation theory such as in the studies of ref. [16]. Equally, one can attempt to estimate the leading $\alpha^{\prime}$ corrections to the fundamental condensate. Such studies could potentially provide a more rigorous test of the correspondence, which does not rely on lattice simulations. It would also be satisfying to understand in more details the suppression of the $\alpha^{\prime}$ corrections in the black hole (deconfined) phase of the D0/D4 system. Finally, one could attempt to study corrections to the probe approximation by taking into account the backreaction of the flavour D4-branes. We leave all of these interesting directions for future work.

\section{Acknowledgments}

S.K. was supported by the Irish Research Council funding. The authors wish to acknowledge the Irish Centre for High-End Computing (ICHEC) for the provision of computational facilities and support (Project Name dsphy003c, dsphy004c and dsphy009c). The support from Action MP1405 QSPACE of the COST foundation is gratefully acknowledged.

\section{A Analytic expression for the condensate susceptibility}

To obtain an expression for the slope of the condensate curve at vanishing bare mass, we will determine the mass dependence of the condensate at small masses. This corresponds to D4-brane embeddings entering the horizon at small angle $\theta_{0}$. Our strategy is to substitute $\theta(\tilde{u})=\theta_{0} \eta(\tilde{u})$ into the equation of motion for $\theta$, derived from the action (3.4) and expand to leading order in $\theta_{0}$. This is equivalent to linearising the equation of motion for $\theta$. The resulting equation for $\eta$ is given by

$$
\eta^{\prime \prime}(u)+\frac{2+5 u^{7}}{u^{8}-u} \eta^{\prime}(u)+\frac{3 u^{5}}{u^{7}-1} \eta(u)=0,
$$

where to simplify the notation we have replaced $\tilde{u} \rightarrow u$. The general solution of equation (A.1) is given by

$$
\eta(u)=C(1){ }_{2} F_{1}\left[1 / 7,3 / 7,4 / 7, u^{7}\right]+C(2) u_{2}^{3} F_{1}\left[4 / 7,6 / 7,10 / 7, u^{7}\right] .
$$

Imposing regularity at the horizon $(u=1)$ fixes one of the integration constants and the solution regular at $u=1$ is given by

$$
\eta(u)=C(1)\left({ }_{2} F_{1}\left[1 / 7,3 / 7,4 / 7, u^{7}\right]-u^{3} \frac{\Gamma(4 / 7)^{2} \Gamma(6 / 7)}{\Gamma(1 / 7) \Gamma(3 / 7) \Gamma(10 / 7)}{ }_{2} F_{1}\left[4 / 7,6 / 7,10 / 7, u^{7}\right]\right) .
$$

The remaining integration constant $C(1)$ can be fixed by imposing $\eta(1)=1$. However, we will not need its value to determine the dependence of the condensate $\tilde{c}$ on the bare mass 
$\tilde{m}$. Indeed, expanding equation (A.3) at large $u$, we obtain

$$
\eta(u)=C(1)\left(\frac{\Gamma(2 / 7) \Gamma(4 / 7)}{\Gamma(3 / 7)^{2}} \frac{1}{u}-\frac{7 \pi \csc (\pi / 7) \Gamma(5 / 7)}{2 \Gamma(1 / 7)^{2} \Gamma(3 / 7)} \frac{1}{u^{3}}+O\left(\frac{1}{u^{5}}\right)\right) .
$$

Note that equation (3.5) can be rewritten as

$$
\theta(u)=\frac{\tilde{m}}{u}+\frac{\tilde{c}}{u^{3}}+O\left(\tilde{m}^{2}\right)+O\left(1 / u^{5}\right),
$$

where we have kept only the terms linear in $\tilde{m}$, since equation (A.4) is valid only to a linear order in $\theta_{0}$. Therefore, to linear order in $\tilde{m}$ we have

$$
\tilde{m}=C(1) \theta_{0} \frac{\Gamma(2 / 7) \Gamma(4 / 7)}{\Gamma(3 / 7)^{2}} ; \quad \tilde{c}=-C(1) \theta_{0} \frac{7 \pi \csc (\pi / 7) \Gamma(5 / 7)}{2 \Gamma(1 / 7)^{2} \Gamma(3 / 7)},
$$

and hence

$$
-\left.\left(\frac{d \tilde{c}}{d \tilde{m}}\right)\right|_{\tilde{m}=0}=-\lim _{m \rightarrow 0}\left(\frac{\tilde{c}}{\tilde{m}}\right)=\frac{7 \pi}{2} \frac{\csc (\pi / 7) \Gamma\left(\frac{3}{7}\right) \Gamma\left(\frac{5}{7}\right)}{\Gamma\left(\frac{1}{7}\right)^{2} \Gamma\left(\frac{2}{7}\right) \Gamma\left(\frac{4}{7}\right)},
$$

which is the result used in equation (3.6).

Open Access. This article is distributed under the terms of the Creative Commons Attribution License (CC-BY 4.0), which permits any use, distribution and reproduction in any medium, provided the original author(s) and source are credited.

\section{References}

[1] J.M. Maldacena, The Large- $N$ limit of superconformal field theories and supergravity, Int. J. Theor. Phys. 38 (1999) 1113 [Adv. Theor. Math. Phys. 2 (1998) 231] [hep-th/9711200] [INSPIRE].

[2] N. Itzhaki, J.M. Maldacena, J. Sonnenschein and S. Yankielowicz, Supergravity and the large- $N$ limit of theories with sixteen supercharges, Phys. Rev. D 58 (1998) 046004 [hep-th/9802042] [INSPIRE].

[3] K.N. Anagnostopoulos, M. Hanada, J. Nishimura and S. Takeuchi, Monte Carlo studies of supersymmetric matrix quantum mechanics with sixteen supercharges at finite temperature, Phys. Rev. Lett. 100 (2008) 021601 [arXiv:0707.4454] [INSPIRE].

[4] S. Catterall and T. Wiseman, Black hole thermodynamics from simulations of lattice Yang-Mills theory, Phys. Rev. D 78 (2008) 041502 [arXiv:0803.4273] [INSPIRE].

[5] M. Hanada, Y. Hyakutake, J. Nishimura and S. Takeuchi, Higher derivative corrections to black hole thermodynamics from supersymmetric matrix quantum mechanics, Phys. Rev. Lett. 102 (2009) 191602 [arXiv:0811.3102] [INSPIRE].

[6] D. Kadoh and S. Kamata, Gauge/gravity duality and lattice simulations of one dimensional SYM with sixteen supercharges, arXiv:1503.08499 [INSPIRE].

[7] V.G. Filev and D. O'Connor, The BFSS model on the lattice, JHEP 05 (2016) 167 [arXiv: 1506.01366] [INSPIRE].

[8] S. Catterall and T. Wiseman, Extracting black hole physics from the lattice, JHEP 04 (2010) 077 [arXiv: 0909.4947] [INSPIRE]. 
[9] M. Hanada, Y. Hyakutake, G. Ishiki and J. Nishimura, Holographic description of quantum black hole on a computer, Science 344 (2014) 882 [arXiv:1311.5607] [INSPIRE].

[10] M. Berkooz and M.R. Douglas, Five-branes in M(atrix) theory, Phys. Lett. B 395 (1997) 196 [hep-th/9610236] [INSPIRE].

[11] T. Banks, W. Fischler, S.H. Shenker and L. Susskind, $M$ theory as a matrix model: A Conjecture, Phys. Rev. D 55 (1997) 5112 [hep-th/9610043] [INSPIRE].

[12] A. Karch and E. Katz, Adding flavor to AdS/CFT, JHEP 06 (2002) 043 [hep-th/0205236] [INSPIRE].

[13] D. Mateos, R.C. Myers and R.M. Thomson, Holographic phase transitions with fundamental matter, Phys. Rev. Lett. 97 (2006) 091601 [hep-th/0605046] [INSPIRE].

[14] D. Mateos, R.C. Myers and R.M. Thomson, Thermodynamics of the brane, JHEP 05 (2007) 067 [hep-th/0701132] [INSPIRE].

[15] V.G. Filev and D. O'Connor, A Computer Test of Holographic Flavour Dynamics, JHEP 05 (2016) 122 [arXiv : 1512.02536] [INSPIRE].

[16] Y. Asano, V.G. Filev, S. Kováčik and D. O'Connor, The flavoured BFSS model at high temperature, JHEP 01 (2017) 113 [arXiv:1605.05597] [INSPIRE].

[17] M. Van Raamsdonk, Open dielectric branes, JHEP 02 (2002) 001 [hep-th/0112081] [INSPIRE].

[18] J. Babington, J. Erdmenger, N.J. Evans, Z. Guralnik and I. Kirsch, Chiral symmetry breaking and pions in nonsupersymmetric gauge/gravity duals, Phys. Rev. D 69 (2004) 066007 [hep-th/0306018] [INSPIRE].

[19] C. Hoyos-Badajoz, K. Landsteiner and S. Montero, Holographic meson melting, JHEP 04 (2007) 031 [hep-th/0612169] [INSPIRE].

[20] I. Kirsch, Generalizations of the AdS/CFT correspondence, Fortsch. Phys. 52 (2004) 727 [hep-th/0406274] [INSPIRE].

[21] T. Albash, V.G. Filev, C.V. Johnson and A. Kundu, A Topology-changing phase transition and the dynamics of flavour, Phys. Rev. D 77 (2008) 066004 [hep-th/0605088] [INSPIRE].

[22] J. Erdmenger, N. Evans, I. Kirsch and E. Threlfall, Mesons in Gauge/Gravity Duals - A Review, Eur. Phys. J. A 35 (2008) 81 [arXiv:0711.4467] [inSPIRE].

[23] Y. Asano and D. O'Connor, Checking gauge/gravity duality with the BFSS model by different lattice discretisations, in preparation. 